\title{
Potential use of Internet of Things to support Life Cycle Assessment of buildings
}

\section{SIGRADI2018 TECHNOPOLITICAS \\ xxii congresso da sociedade iberoamericana de gráfica digital 22th conference of the iberoamerican society of digital graphics $07|08| 09 \mid$ novembro|2018 iau usp | são carlos | sp br}

\author{
Natália Nakamura Barros \\ Universidade Estadual de Campinas | Brazil | natalianakamura.arq@gmail.com \\ Regina Coeli Ruschel \\ Universidade Estadual de Campinas | Brazil | ruschel@g.unicamp.br
}

\begin{abstract}
This article summarize the initial discoveries of doctoral research, whose the principal aim is to analyze the use of Internet of Things to support Life Cycle Assessment of buildings. The first cycle of this thesis consists a preliminary investigation on electronics newspapers that deal the integration LCA and IOT. The results reveals IoT technology could provide real-time data collection, possibility of Big Data collection and monitoring, and greater precision and reliability of data. IoT-Based LCA is very promissory and innovative. In this way, this research intends to bring a relevant contribution to architecture, engineering and construction (AEC).
\end{abstract}

Keywords: Life cycle assessment; Internet of Things; LCA; IOT.

\section{INTRODUÇÃO}

A necessidade de redução dos impactos ambientais é crescente em todo o mundo. As atividades da construção civil principalmente geram um alto impacto ambiental. A Avaliação do Ciclo de Vida - ACV constitui a metodologia preferida internacionalmente para investigar sistematicamente os impactos de um produto, processo ou sistema em cada etapa de seu ciclo de vida, desde a extração da matéria-prima até o fim-de-vida (Guinée, 2002).

A ACV é dividida em quatro fases, sendo estas: definição do objetivo e escopo, análise de inventário, avaliação de impacto e interpretação (ABNT, 2009b). A etapa de análise de inventário, na qual ocorre a coleta de dados, pode ser trabalhosa e demorada (Rist, 2011), uma vez que há uma grande quantidade de dados requeridos para a avaliação (Mashhadi \& Behdad, 2017). Além disso, à medida que os dados são coletados e se amplia o conhecimento sobre o sistema, novos requisitos ou limitações podem ser identificados, requerendo mudança nos procedimentos (ABNT, 2009b).

Atualmente, a forma tradicional de coleta de dados ainda é manual, e o processo de avaliação busca dados em bases existentes (Tao, Zuo, Xu, Lv, \& Zhang, 2014), o que diminui a confiabilidade e precisão dos mesmos. Deste modo, os principais problemas da ACV relacionamse principalmente à disponibilidade e à qualidade dos dados (Reap, Roman, Duncan, \& Bras, 2008).

A quantidade de informações e a complexidade que envolve a ACV de edificações fazem com que métodos computacionais e inovadores sejam necessários (Schulueter \& Thesseling, 2009). Dentre as tecnologias mais inovadoras, está a Internet das Coisas - IoT, na qual a Internet é usada como uma plataforma global para máquinas e objetos inteligentes comunicarem, dialogarem, compilarem e dialogarem (Miorandi, Sicari, De Pellegrini, \& Chlamtac, 2012). A loT cria um tecido de rede inteligente e invisível que pode ser detectado, controlado e programado (Chase, 2013), no qual entidades físicas e digitais podem ser vinculadas, para permitir uma nova classe de aplicativos e serviços (Miorandi et al., 2012).

As práticas da loT estão se espalhando para diversos domínios de ciência e tecnologia, entretanto atualmente não se tem conhecimento de como aplicá-la à ACV de edificações. Deste modo, questiona-se: a loT consegue apoiar a totalidade dos dados necessários à ACV de edificações?

O presente artigo tem como meta revelar as descobertas iniciais de uma pesquisa de doutorado, cujo objetivo principal é verificar o uso de Internet das Coisas para apoiar a Avaliação do Ciclo de Vida de edificações. Para tal, foi realizada uma pesquisa bibliográfica em periódicos relevantes da área a fim de explicitar os estudos que tratam da integração entre ACV e IoT.

\section{FUNDAMENTAÇÃO}

Esta seção fundamentará Avaliação do Ciclo de Vida e Internet das Coisas.

\section{AVALIAÇÃO DO CICLO DE VIDA}

A avaliação de ciclo de vida é uma técnica para contabilização de impactos de produtos e processos desde a extração de matéria-prima, passando por produção, uso e disposição final (ASSOCIAÇÃO BRASILEIRA DE NORMAS TÉCNICAS, 2009b). O amadurecimento de pesquisas e aplicações da ACV nos últimos 10 anos, principalmente, torna-a um apoio 
fundamental na estimativa de impactos e cálculo de indicadores ambientais

A Society of Environmental Toxicology and Chemistry SETAC foi o primeiro organismo internacional a trabalhar no desenvolvimento da ACV, e foi posteriormente normalizada pela International Standardization Organization - ISO (Guinée, 2002). No Brasil, a ACV foi normatizada pela NBR ISO 14040: gestão ambiental, avaliação do ciclo de vida - princípios e estrutura (ABNT, 2009a) e NBR ISO 14044: gestão ambiental, avaliação do ciclo de vida - requisitos e orientações, que divide a ACV em quatro fases, sendo estas: objetivo e definição do escopo, análise de inventário, avaliação de impacto e interpretação (ABNT, 2009b).

Uma das etapas mais importantes da ACV é a análise de inventário do ciclo de vida, que envolve a coleta de dados e procedimentos de cálculo para quantificar as entradas e saídas pertinentes de um sistema (ABNT, 2009b). A tarefa mais exigente na realização de uma ACV é a coleta de dados. Embora uma grande quantidade de dados esteja disponível na literatura, alguns processos e materiais ainda não estão disponíveis (Goedkoop, Oele, Leijting, Ponsioen, \& Meijer, 2016). Deste modo, a coleta de dados pode ser um processo de intensiva demanda de recursos (ABNT, 2009b).

Os principais desafios da aplicabilidade da ACV no contexto brasileiro são principalmente devido à ausência de base de dados nacional de materiais de construção. Uma alternativa à esta ausência é o uso de um banco de dados internacional, porém devido às enormes diferenças em termos de tecnologias, geologia, clima, densidade de população, biomas, tipos de produtos, entre outros; a aplicação destas bases em países que não possuem um banco de dados torna-se um desafio (Bauman \& Tillman, 2004). Desse modo, o levantamento de informações acerca dos materiais de construção pode ser complexo, demorado e oneroso.

Outro grande desafio, é a falta de uma descrição detalhada do procedimento da ACV nas normas ISO, o que torna difícil avaliar se foi feita de acordo com o padrão. Deste modo, é necessária a documentação cuidadosa da meta, questões de escopo e interpretação da ACV, para comprovar sua adequação às normas ISO. Outra possibilidade é a inclusão de uma revisão de pares por peritos independentes (Goedkoop et al., 2016).

As principais aplicações da ACV são: verificação de oportunidades de melhorias a partir da identificação dos principais impactos ambientais no ciclo de vida de um produto; análise da contribuição das fases do ciclo de vida do produto para a carga ambiental global; comparação entre produtos para comunicação interna ou externa; base para as declarações de produtos ambientais e métricas padronizadas; e identificação de indicadores de desempenho de produtos (Goedkoop et al., 2016).

\section{INTERNET DAS COISAS}

A IOT refere-se a um ambiente de computação hiperconectado, independentemente da natureza das coisas conectadas. Em outras palavras, é uma réplica virtual do mundo físico. As fronteiras entre os mundos virtual, físico e biológico tornam-se, assim, quase inexistentes, criando-se novos sistemas cyber-físicos. Não apenas os seres humanos e outros organismos vivos, mas virtualmente qualquer objeto, animado ou inanimado, podem ser conectados à $10 T$ e conversar uns com os outros através de sensores e conectividade sem fio, rastreados em tempo real (Ozdemir \& Hekim, 2018).

A definição de Internet of Things ainda é imprecisa e pode ter diferentes facetas dependendo da perspectiva adotada, como, por exemplo, ela pode ser: orientada para as coisas, orientada para a Internet e orientada à semântica (Atzori, lera, \& Morabito, 2010). Na primeira perspectiva, é razoável definir a loT como: coisas que têm identidades e personalidades virtuais operando em espaços inteligentes usando interfaces inteligentes para se conectar e se comunicar dentro de contextos sociais, ambientais e de usuários. Na segunda perspectiva, orientada para a Internet, coloca-se o foco na integração contínua, e pode ser formulada como: objetos interconectados tendo um papel ativo no que pode ser chamado de Internet do Futuro. Já na perspectiva semântica, IoT significa uma rede mundial de objetos interconectados exclusivamente endereçáveis, baseada em protocolos de comunicação padrão (European Comission, 2008).

A evolução da Internet começa com a conexão de dois computadores e depois com a criação da World Wide Web, em 1991, conectando um grande número de computadores. A Internet móvel surgiu conectando dispositivos móveis à Internet. Com o crescente uso das redes sociais, as identidades das pessoas se juntaram à Internet, e finalmente, há um movimento contínuo em direção à Internet das Coisas, conectando todos os objetos do dia-a-dia à Internet (Perera, Zaslavsky, Christen, \& Georgakopoulos, 2014).

A frase Internet of Things foi cunhada há 10 anos pelos fundadores do MIT Auto-ID Center, com menção especial a Kevin Ashton, em 1999, e David L. Brock, em 2001. O termo Auto-ID refere-se a qualquer classe ampla de tecnologias de identificação usadas na indústria para automatizar, reduzir erros e aumentar a eficiência. Essas tecnologias incluem códigos de barras, cartões inteligentes, sensores, reconhecimento de voz e biometria. Mas desde 2003, a principal tecnologia Auto-ID tem sido a identificação por radiofrequência - RFID (European Comission, 2010).

Os sistemas RFID podem ser usados para monitorar objetos em tempo real, isso permite mapear o mundo real no mundo virtual. Do ponto de vista físico, uma tag RFID é um pequeno microchip conectado a uma antena (Atzori et al., 2010). Quando conectados às redes de sensores, os sistemas RFID permitem um melhor acompanhamento do status das coisas, ou seja, sua localização, temperatura, movimentos, etc (Atzori et al., 2010).

Existem três categorias de redes de sensores que compõem a IOT: redes de sensores corporais, redes de sensores de objetos e redes de sensores de ambiente (Perera et al., 2014). A maioria das redes de sensores implantadas, hoje, é sem fio, baseadas em diversas tecnologias, como: rede de área pessoal sem fio, por exemplo, Bluetooth; rede local sem fio, por exemplo, Wi- 
$\mathrm{Fi}$; rede sem fio de área metropolitana; rede de longa distância sem fio, por exemplo, redes $2 \mathrm{G}$ e $3 \mathrm{G}$; e rede de satélites (Perera et al., 2014).

A arquitetura loT pode ser composta por 4 principais etapas (Bradicich, 2017):

i) Etapa 1: Coisas são conectadas a sensores para captura de dados e atuadores através de conexão com ou sem fio;

ii) Etapa 2: Sensores e atuadores se conectam a gateways, comutadores e sistemas de aquisição de dados;

iii) Etapa 3: Sistemas de TI calculam estes dados, fornecem acesso à aplicativos e revelam insights sobre as coisas conectadas, os dispositivos e o ambiente ao redor;

iv) Etapa 4: Centro de dados remoto ou a nuvem.

Estas etapas são realizadas em um middleware, que consiste em uma camada de software ou um conjunto de subcamadas interpostas entre os níveis tecnológico e de aplicação. O middleware deve incluir funções relacionadas ao gerenciamento, privacidade e segurança de todos os dados trocados (Atzori et al., 2010). As arquiteturas de middleware propostas nos últimos anos para a IoT geralmente seguem a abordagem Service Oriented Architecture - SOA, composta pelas seguintes camadas:

i) Abstração de objetos: camada capaz de harmonizar - acesso aos diferentes dispositivos com uma linguagem e um procedimento comuns;

ii) Gerenciamento de serviços: fornece as principais funções que devem estar disponíveis para cada objeto e permite o gerenciamento no cenário de $I 0 T$;

iii) Composição do serviço: criação de processos complexos com uma sequência de ações coordenadas;

iv) Aplicações: exportação de todas as funcionalidades do sistema para o usuário final (Atzori et al., 2010).

O uso de redes de sensores tem sido proposto em diversos cenários de aplicação, como monitoramento ambiental, e-health, sistemas de transporte inteligentes, militares e monitoramento de plantas industriais (Atzori et al., 2010). Além destas, loT pode ser utilizada na área de varejo, logística, alimentos, saúde, ambientes inteligentes, eficiência de recursos, poluição e prevenção de desastres (European Comission, 2008).

Os principais desafios da $l o T$ relacionam-se à ausência de governança, privacidade e segurança (European Comission, 2008). A automação extrema representa vulnerabilidades que foram pouco consideradas até agora. Primeiro, os sistemas altamente integrados são vulneráveis a riscos sistêmicos, como o colapso total da rede no caso de falha de uma de suas partes, por exemplo, por vírus de hackers, que podem invadir totalmente os sistemas integrados. Em segundo lugar, conectividade extrema pode criar novas estruturas de poder social e político (Ozdemir \& Hekim, 2018).

\section{METODOLOGIA}

A metodologia utilizada nesta primeira etapa da tese, consistiu em pesquisa bibliográfica (Marconi \& Lakatos, 2010), realizada em periódicos eletrônicos a fim de evidenciar os artigos que tratam da integração entre ACV e IOT. A pesquisa foi realizada em junho de 2018 e buscou a combinação entre as palavras-chave: LCA AND IoT; Life Cycle Assessment AND Internet of Things, nas bases de dados eletrônicas relevantes para AEC: Scopus, ASCE, Web of Science e Enginnering Village. A tabela 1 mostra o protocolo desta pesquisa bibliográfica.

Tabela 1: Protocolo de Pesquisa Bibliográfica: ACV + IoT.

\begin{tabular}{|c|c|}
\hline $\begin{array}{ll}\text { Data } & \text { da } \\
\text { Pesquisa } & \end{array}$ & 19/06/2018 \\
\hline $\begin{array}{l}\text { Termos } \\
\text { busca }\end{array}$ & $\begin{array}{l}\text { LCA + IoT; "Life Cycle Assessment" AND } \\
\text { "Internet of Things" }\end{array}$ \\
\hline Fonte & $\begin{array}{l}\text { SCOPUS - https://www.scopus.com/; } \\
\text { COMPENDEX } \\
\text { www.engineeringvillage.com/; } \\
\text { ASCE LIBRARY - ascelibrary.org/; } \\
\text { WEB OF SCIENCE } \\
\text { webofknowledge.com/. }\end{array}$ \\
\hline Idioma & Inglês \\
\hline $\begin{array}{l}\text { Critérios } \\
\text { Pesquisa }\end{array}$ & $\begin{array}{l}\text { Scopus: Título, Resumo e Palavra-Chave } \\
\text { ASCE: Anywhere } \\
\text { Web of Science: Topic } \\
\text { Enginnering Village: All Fields }\end{array}$ \\
\hline $\begin{array}{l}\text { Critérios } \\
\text { exclusão }\end{array}$ & $\begin{array}{l}\text { Foram excluídos artigos que não tratam } \\
\text { sobre a integração entre ACV e IOT; } \\
\text { Foram excluídos artigos cujos termos de } \\
\text { busca tem outro significado senão: IOT= } \\
\text { Internet of Things e LCA = Life Cycle } \\
\text { Assessment }\end{array}$ \\
\hline
\end{tabular}

Fonte: elaborado pelo autor.

\section{RESULTADOS}

A pesquisa bibliográfica realizada resultou em seis artigos relevantes que tratam de $I O T$ e ACV. Pesquisadores estão discutindo o potencial uso de $10 T$ na ACV (Mashhadi \& Behdad, 2017) e em diversas aplicações, como: no gerenciamento de resíduos de equipamentos elétricos e eletrônicos (Gu, Ma, Guo, Summers, \& Hall, 2017), na etapa de fabricação de sistemas solares (Tu, Chung, Chiu, Chung, \& Tzeng, 2017), para minimizar o consumo de energia (Arshad, Zahoor, Shah, Wahid, \& Yu, 2017; Tao et al., 2014; Zuo, Tao, \& Nee, 2018). Porém, há poucos estudos que tratam da aplicação de Internet das Coisas na ACV com foco em edificações.

O crescimento da Industria 4.0 pode impor novas mudanças e restrições para ACV (Mashhadi \& Behdad, 2017). A tecnologia IoT pode proporcionar a coleta de dados de consumo de energia e impacto ambiental em tempo real, inteligente e dinâmica, abrangendo a multiestrutura (ex: desde partes e componentes do produto) e multi-níveis (ex: projeto, fabricação, transporte, uso e reciclagem) da avaliação de todo o ciclo de vida do produto (Tao et al., 2014).

No quadro conceitual de ACV, cada máquina ou processo de fabricação teria um agente de rastreamento ambiental interconectado que registra e relata inputs e outputs em 
tempo real. Os protocolos de comunicação como iv) MTconnect e RFID podem facilitar a gravação de dados, de modo que os dados possam ser processados em uma unidade de processamento (Mashhadi \& Behdad, 2017).

Tu et al (2017) propuseram uma nova abordagem de gerenciamento e estimativa de pegada de carbono baseado em IoT, aplicado durante a etapa de fabricação de painéis fotovoltaicos. Para tal, utilizaram o EPCglobal network como plataforma da tecnologia IOT por suportar $v$ ) as operações lógicas baseadas em RFID.

\section{ARQUITETURA IOT BASEADA EM ACV}

A arquitetura de $10 T$ baseada na ACV, com foco em consumo de energia, proposta por Zuo, Tao e Nee vi) (2018), pode ser constituída pelas camadas de:

i) Camada de coleta de serviços: agrupa métodos, algoritmos, informações de consumo de energia do material, processo de fabricação, peça padrão, etc., dos provedores e documenta-os em serviços padronizados, que são registrados e depositados na nuvem.

ii) Camada Nuvem: é o ambiente de troca de dados abrangente e padronizado que mantém toda a informação e conhecimento ao longo de todo o ciclo de vida do produto;

iii) Camada de gerenciador de serviços: fornece funções ou serviços para os usuários gerenciarem, acessarem e solicitarem serviços;

Tabela 2: Estrutura de Internet das Coisas baseada em Avaliação de Ciclo de Vida.

\begin{tabular}{|c|c|c|}
\hline Camadas & Sub-Camadas & Exemplos \\
\hline \multirow[t]{3}{*}{ Camada de Percepção } & Atividades de Fabricação & $\begin{array}{l}\text { Processo (Torno, Máquinas, Equipamentos, Operador) } \\
\text { Workshop (Materiais, Pessoal) } \\
\text { Linhas de Produção (Equipamentos de Computação) }\end{array}$ \\
\hline & Coleta & $\begin{array}{l}\text { Medidor inteligente } \\
\text { RFID } \\
\text { Sensores de Fibra ótica }\end{array}$ \\
\hline & Comunicação & $\begin{array}{l}2 \mathrm{G} / 3 \mathrm{G} / 4 \mathrm{G} \\
\text { Wireless } \\
\text { Satellite }\end{array}$ \\
\hline \multirow[t]{3}{*}{ Camada de Dados } & Dados de avaliação & $\begin{array}{l}\text { Indicadores ambientais } \\
\text { Indicadores de consumo de recursos }\end{array}$ \\
\hline & $\begin{array}{ll}\text { Dados } & \text { básicos do } \\
\text { empreendimento } & \end{array}$ & $\begin{array}{l}\text { Usuários } \\
\text { Materiais } \\
\text { Processos } \\
\text { Equipamentos }\end{array}$ \\
\hline & $\begin{array}{l}\text { Dados de todo o ciclo de vida } \\
\text { do produto }\end{array}$ & Sistema \\
\hline \multirow[t]{3}{*}{ Camada de serviços } & Sistemas de serviços básicos & $\begin{array}{l}\text { Gerenciamento de usuários } \\
\text { Gerenciamento de funções } \\
\text { Entrada e atualização de dados }\end{array}$ \\
\hline & $\begin{array}{lll}\text { Serviços } & \text { Computacionais } & \text { e } \\
\text { cálculos } & \end{array}$ & $\begin{array}{l}\text { Modelo de partes do ciclo de vida } \\
\text { Cálculo da caracterização e normalização } \\
\text { Cálculo do impacto ambiental }\end{array}$ \\
\hline & Serviços de análise e avaliação & $\begin{array}{l}\text { Avaliação ambiental } \\
\text { Avaliação das partes } \\
\text { Avaliação ponderada } \\
\text { Análise da estrutura } \\
\text { Análise do potencial de economia de energia } \\
\text { Análise dos resultados da avaliação }\end{array}$ \\
\hline Camada de Aplicações & Usuários & $\begin{array}{l}\text { Empreendedores } \\
\text { Governantes } \\
\text { Pessoas } \\
\text { Outros }\end{array}$ \\
\hline
\end{tabular}

Fonte: traduzido e adaptado de Tao et al (2014)
Camada de usuários: são definidos como os interessados que exigem serviços. Os usuários são conectados à nuvem por meio de interfaces de usuário baseadas em navegador, que coletam várias solicitações de serviço dos usuários e as documentam em um formato padronizado. As solicitações de serviço documentadas são então entregues à camada do gerenciador de serviços.

Camada loT: é responsável por detectar o processo do ciclo de vida do produto físico, permitindo que eles estejam conectados à rede mais ampla e coletando e processando os dados;

Camada de ciclo de vida de fabricação física: contém o ambiente físico e as atividades físicas realizadas em todo o ciclo de vida do produto.

Em suma, a arquitetura de loT baseada em ACV possui 4 camadas principais: (i) percepção, que consiste na coleta de dados em tempo-real, através de RFID, wireless, mobile e sensores, embedded object logic, objeto ad-hoc networking, e infraestrutura de informação baseada na internet; (ii) dados; (iii) serviços, que consistem no serviços básicos, computacionais e de análise e avaliação e; (iv) aplicação, que consiste no fornecimento de dados da avaliação para diferentes usuários (Tao et al., 2014) conforme explicita a tabela 2. 


\section{PRINCIPAIS BENEFÍCIOS}

A intensa interconexão fornecida pela Internet das Coisas permite que os avatares do cyber-espaço das máquinas sejam marcados e monitorados, bem como, permite acompanhar quaisquer entradas ou saídas, e avaliar os impactos correspondentes, individualmente e em tempo real. A rastreabilidade fornecida pela $10 T$ deve permitir que as ACVs futuras possam avaliar os impactos ambientais em qualquer nível em relação aos processos, produtos, regiões ou durações (Mashhadi \& Behdad, 2017).

Os benefícios do uso da IoT na ACV de um modelo de painel fotovoltaico de single-silicon produzido em Taiwan, foram comprovados por Tu et al (2017). Os resultados da pesquisa revelaram que houve uma significativa redução do tempo e custo de trabalho durante a coleta de dados e auditoria da ACV, além da melhoria na qualidade e precisão dos dados.

\section{PRINCIPAIS APLICAÇÕES}

A IOT está revolucionando as atividades da vida diária, monitorando diferentes cenários e decisões inteligentes para melhorar o estilo de vida e proteger o meio ambiente. Existem numerosas aplicações da loT na vida diária, dentre as que se relacionam à área de $\mathrm{AEC}$ estão (Arshad et al., 2017):

$>$ Smart Homes: as tecnologias loT podem ser utilizadas em diversas situações para monitorar as atividades dos habitantes e tomar decisões que podem, por exemplo, poupar energia;

> Transporte: possibilidade de rastreamento de veículos e produtos que utilizam RFIDs e sensores, da fonte para destino em tempo real;

$>$ Cidades inteligentes: a cidade é uma combinação de diferentes domínios inteligentes como transporte inteligente, mecanismo inteligente de economia de energia, segurança inteligente e muito mais.

\section{DISCUSSÃO}

Esta pesquisa revelou que a tecnologia $l o T$ pode apoiar a ACV de edificações, proporcionando a coleta de dados de consumo de energia e impacto ambiental em tempo real, inteligente e dinâmica, abrangendo a multiestrutura, desde partes e componentes do produto, até multi-níveis, como no projeto, fabricação, transporte, uso e reciclagem. Além disso, o uso da loT na ACV pode reduzir significativamente o tempo e custo de trabalho durante a coleta de dados e auditoria da ACV, e melhorar a qualidade e precisão dos dados.

O desenvolvimento de novas tecnologias está revolucionando e automatizando todos os setores da atividade humana. Internet das Coisas já é uma realidade ímpar presente no dia-a-dia das pessoas, principalmente com o constante uso de celular. Deste modo, IoT pode também ser utilizada para facilitar a Avaliação de Ciclo de Vida, e o presente estudo mostrou que são inúmeros os benefícios desta integração.
A integração de $10 T$ e ACV de edificações pode trazer inúmeros benefícios para todos os agentes envolvidos na construção civil sustentável. A possibilidade de obtenção de resultados rápidos e confiáveis sobre o desempenho ambiental dos edifícios, pode auxiliar tanto o especialista ACV, gestores de facilities e simuladores de desempenho energético, quanto os projetistas, durante a tomada de decisão e opção por estratégias soluções mais sustentáveis.

O uso de tecnologias $10 T$, para a percepção inteligente em tempo real e a coleta de dados de impacto ambiental gerados com ênfase na edificação, agrega conhecimento para a temática de cidades sustentáveis e inteligentes. A rastreabilidade fornecida pela $10 T$ deve permitir que as ACVs futuras possam avaliar os impactos ambientais em qualquer nível a em relação aos processos, produtos, regiões ou durações. Além disso, o suporte inteligente à ACV de edificações é algo extremamente inovador.

O uso de Internet das Coisas para apoiar a Avaliação do Ciclo de Vida de edificações é muito promissor e inovador. Entretanto, percebe-se que os estudos nesta área ainda são muito abrangentes, e são necessários estudos mais específicos de como esta integração pode ser realizada, principalmente voltada ao setor de arquitetura, engenharia e construção. Quanto à difusão do conhecimento, esta pesquisa pode ser usada como referência e apoio à geração de conhecimento em campos práticos e teóricos.

\section{REFERÊNCIAS}

ABNT, A. B. de N. T. (2009a). NBR ISO 14040: Gestão ambiental - Avaliação do ciclo de vida - Princípios e estrutura. Rio de Janeiro.

ABNT, A. B. de N. T. (2009b). NBR ISO 14044: Gestão ambiental - Avaliação do ciclo de vida - Requisitos e orientações. Rio de Janeiro.

Arshad, R., Zahoor, S., Shah, M. A., Wahid, A., \& Yu, H. (2017). Green IoT: An Investigation on Energy Saving Practices for 2020 and Beyond. IEEE Access, 5, 15667-15681. https://doi.org/10.1109/ACCESS.2017.2686092

Atzori, L., Iera, A., \& Morabito, G. (2010). The Internet of Things: A survey. Computer Networks, 54(15), 2787-2805. https://doi.org/10.1016/J.COMNET.2010.05.010

Bauman, H., \& Tillman, A.-M. (2004). The Hitch Hiker's Guide to LCA (1st ed.). Estados Unidos: Lightning Source.

Bradicich, T. (2017). What the intelligent edge is and why it's us eful | HPE | HPE ${ }^{\mathrm{TM}}$. Retrieved March 20, 2018, from https://www.hpe.com/us/en/insights/articles/the-intelligentedge-what-it-is-what-its-not-and-why-its-us eful-1704.html

Chase, J. (2013). The evolution of the Internet of Things. Texas Instrum., p. p.7.

European Comission. (2008). Internet of Things in 2020: Roadmap for the Future.

European Comission. (2010). Vision and Challenges for Realising the Internet of Things. Luxembourg: Publications Office of the European Union.

Goedkoop, M., Oele, M., Leijting, J., Ponsioen, T., \& Meijer, E. (2016). Introduction to LCA with SimaPro (5.2). California, USA.

Gu, F., Ma, B., Guo, J., Summers, P. A., \& Hall, P. (2017). Internet of things and Big Data as potential solutions to the
5

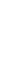


problems in waste electrical and electronic equipment management: An exploratory study. Waste Management, 68 , 434-448. https://doi.org/10.1016/j.wasman.2017.07.037

Guinée, J. B. (2002). Handbook on life cycle assessment. Operational guide to the ISO standards. Dordrecht: Kluwer Academic Publishers.

Marconi, M. de A., \& Lakatos, E. M. (2010). Fundamentos de metodologia científica (7th ed.). São Paulo: Atlas.

Mashhadi, A. R., \& Behdad, S. (2017, December). Ubiquitous Life Cycle Assessment (U-LCA): A Proposed Concept for Environmental and Social Impact Assessment of Industry 4.0. Manufacturing Letters. https://doi.org/10.1016/j.mfglet.2017.12.012

Miorandi, D., Sicari, S., De Pellegrini, F., \& Chlamtac, I. (2012). Internet of things: Vision, applications and research challenges. Ad Hoc Networks, 10(7), 1497-1516. https://doi.org/10.1016/J.ADHOC.2012.02.016

Ozdemir, V., \& Hekim, N. (2018). Birth of Industry 5.0: Making Sense of Big Data with Artificial Intelligence, "'The Internet of Things"' and Next-Generation Technology Policy. Journal of Integrative Biology, 22(1). https://doi.org/DOI: 10.1089/omi.2017.0194

Perera, C., Zaslavsky, A., Christen, P., \& Georgakopoulos, D. (2014). Context Aware Computing for The Internet of Things: A Survey. IEEE Communications Surveys \& Tutorials, 16(1), 414-454. https://doi.org/10.1109/SURV.2013.042313.00197
Reap, J., Roman, F., Duncan, S., \& Bras, B. (2008). A survey of unresolved problems in life cycle assessment. Int $J$ Life Cycle Assess, (13), 290-300. https://doi.org/10.1007/s11367-008-0008-x

Rist, T. (2011). A path to BIM-based LCA for whole-buildings. Norweigan University of Science and Technology, Spring.

Schulueter, A., \& Thesseling, F. (2009). Building information model based energy/exergy performance assessment in early design stages. Automation in Construction, 18(2), 153-163.

Tao, F., Zuo, Y., Xu, L. Da, Lv, L., \& Zhang, L. (2014). Internet of things and BOM-Based life cycle assessment of energysaving and emission-reduction of products. IEEE Transactions on Industrial Informatics, 10(2), 1252-1261. https://doi.org/10.1109/TII.2014.2306771

Tu, M., Chung, W. H., Chiu, C. K., Chung, W., \& Tzeng, Y. (2017). A novel IoT-based dynamic carbon footprint approach to reducing uncertainties in carbon footprint assessment of a solar PV supply chain. In 2017 4th International Conference on Industrial Engineering and Applications (ICIEA) (pp. 249-254). Institute of Electrical and Electronics Engineers Inc. https://doi.org/10.1109/IEA.2017.7939216

Zuo, Y., Tao, F., \& Nee, A. Y. C. (2018). An Internet of things and cloud-based approach for energy consumption evaluation and analysis for a product. International Journal of Computer Integrated Manufacturing, 31(4-5), 337-348. https://doi.org/10.1080/0951192X.2017.1285429 